Mappemonde

Revue trimestrielle sur l'image géographique et les formes du territoire

$120 \mid 2017$

Varia

\title{
Enjeux de l'information territoriale locale ouverte et participative
}

\author{
Marianne Guérois et Malika Madelin
}

\section{OpenEdition}

Journals

Édition électronique

URL : http://journals.openedition.org/mappemonde/2847

DOI : $10.4000 /$ mappemonde.2847

ISSN : 1769-7298

Éditeur

UMR ESPACE

Référence électronique

Marianne Guérois et Malika Madelin, « Enjeux de l'information territoriale locale ouverte et participative », Mappemonde [En ligne], 120 | 2017, mis en ligne le 01 avril 2017, consulté le 25 septembre 2020. URL : http://journals.openedition.org/mappemonde/2847 ; DOI : https://doi.org/ 10.4000/mappemonde.2847

Ce document a été généré automatiquement le 25 septembre 2020.

\section{cc) (1)}

La revue Mappemonde est mise à disposition selon les termes de la Licence Creative Commons Attribution - Pas d'Utilisation Commerciale - Partage dans les Mêmes Conditions 4.0 International. 


\title{
Enjeux de l'information territoriale locale ouverte et participative
}

\author{
Marianne Guérois et Malika Madelin
}

1 Ce dossier est une invitation à explorer les enjeux de l'information territoriale locale ouverte et participative. Cette proposition s'appuie initialement sur des questions discutées lors de séminaires et d'ateliers animés par l'axe Information Territoriale Locale du Collège International des Sciences du Territoire (CIST). La création d'un axe propre à l'échelon local répond à la nécessité d'y voir plus clair dans les enjeux soulevés par la multiplication récente de données locales de natures très hétérogènes. L'origine de cette profusion d'informations est souvent associée aux puissantes mutations technologiques qui concourent à la collecte de données localisées de plus en plus précises et massives (mais pas toujours plus accessibles) et qui renvoient aussi bien aux résolutions très fines des nouveaux capteurs de l'imagerie satellitaire, à l'intégration d'outils de localisation aux objets du quotidien (de téléphonie mobile notamment) qu'à la multiplication des plateformes du web invitant les internautes à partager leurs localisations (des réseaux sociaux aux plateformes de vente ou d'échange de services). Plus largement, la rencontre entre les nouvelles technologies de localisation et le mouvement des sciences participatives est en train de renouveler largement la collecte volontaire d'information locale par des individus, dans un contexte de décentralisation de la production et de l'usage des données. Par ailleurs, les dispositions législatives favorables à l'ouverture des données publiques contribuent à la diffusion de données institutionnelles de nature certes plus conventionnelle (qu'il s'agisse de données censitaires, administratives, de données de localisation d'équipements ou encore d'enquêtes), mais dont l'accès était jusqu'à présent relativement limité. Au-delà de ce constat, il reste malgré tout difficile de définir la notion d'information territoriale locale de manière univoque, tant l'identification de ce qui relève de l'échelon local peut varier selon les problématiques envisagées, se référant tantôt à la rue, tantôt à la parcelle, ou bien à toute maille administrative infrarégionale... On pourrait être tentés d'en circonscrire plus nettement les contours en l'associant à la question de la finesse de la résolution spatiale, mais la référence technique au niveau de détail le plus fin est loin d'être la plus pertinente pour saisir les phénomènes locaux qui font sens pour les 
individus. Plus largement, l'information territoriale locale peut sans doute être mieux appréhendée par ses liens avec les territoires de la proximité, de l'observation directe et du quotidien, que ce soit en termes de collecte ou d'usage des données.

La profusion récente de données locales soulève des questionnements qui dépassent les thématiques et les objets d'étude abordés, tant en termes de production, de traitement que d'usages de l'information. Les trois premières contributions proposées pour amorcer le dossier apportent des éclairages importants sur certaines de ces questions à partir de versions remaniées et approfondies de communications scientifiques qui ont été présentées lors de la session Information territoriale du $2^{\mathrm{e}}$ colloque du CIST (27-29 mars 2014).

En interrogeant la légitimité et la qualité des données citoyennes sur la biodiversité, $\mathrm{X}$. Amelot, $\mathrm{L}$. Couderchet et $\mathrm{M}$. Noucher s'intéressent à la manière dont l'essor des données contributives a transformé l'observation de la biodiversité en France. À partir de l'analyse croisée de deux projets emblématiques, la base des ZNIEFF et l'Atlas de la faune en Aquitaine, ils revisitent l'opposition fréquente entre données officielles et données contributives, en s'interrogeant sur les critères de validité et de robustesse de ces dernières ainsi que sur leur recevabilité par les politiques de conservation de la biodiversité (https://journals.openedition.org/mappemonde/2861).

4 Le deuxième article porte sur les outils de traitement et de représentation des données territoriales permettant d'animer le débat public. Dans le domaine de l'observation territoriale, la gestion de l'information spatialisée impose en efFet de mener une réflexion non seulement sur l'harmonisation de données provenant de sources hétérogènes, mais aussi sur les formes de la restitution de cette information auprès de plusieurs usagers potentiels (politiciens, chercheurs, praticiens, citoyens). Sur la base de l'expérience acquise dans le cadre du programme européen ESPON, R. Ysebaert, I. Salmon, B. Le Rubrus et C. Bernard présentent un modèle générique d'organisation, de partage et de diffusion d'informations régionales qui répond à des difficultés fréquemment rencontrées lors du montage d'observatoires régionaux et lors de la coordination de producteurs de données multiples (https:// journals.openedition.org/mappemonde/2932).

5 La troisième contribution vient alimenter la rubrique Lieux et porte sur l'usage des données de télédétection dans un contexte de gestion d'une catastrophe naturelle. C. Dubois, H. de Boissezon et P. Escudier proposent un retour d'expérience sur la création d'une base de données territoriales suite au tremblement de terre qui a frappé Haïti en 2010. Les auteurs montrent la complexité de la transformation de données de télédétection en ressources pour les acteurs locaux, en distinguant deux moments spécifiques de production de l'information locale : un contexte d'urgence, qui a mis au premier plan l'organisation territorialisée des secours quelques jours après la catastrophe, et un contexte de reconstruction, qui soulève la question de l'articulation de l'expertise technico-scientifique avec les expériences situées des populations d'Haïti.

6 Au-delà de ces premières contributions, nous espérons que l'ouverture de ce dossier permettra de confronter des travaux portant sur les nombreux aspects théoriques, méthodologiques et techniques de l'essor des données ouvertes et participatives (choix des échelons d'analyse pertinents pour des données élémentaires de résolution très fine, croisement de données thématiques de granulométrie ou de géométries difFérentes, articulation entre données d'enquête et données contextuelles, traitement et visualisation de phénomènes dont la nature discontinue ressort fortement à 
l'échelon local, etc.), dans un contexte amené à modifier en profondeur les relations entre chercheurs, citoyens et acteurs du développement territorial.

\section{AUTEURS}

MARIANNE GUÉROIS

Université Paris Diderot

MALIKA MADELIN

Université Paris Diderot 